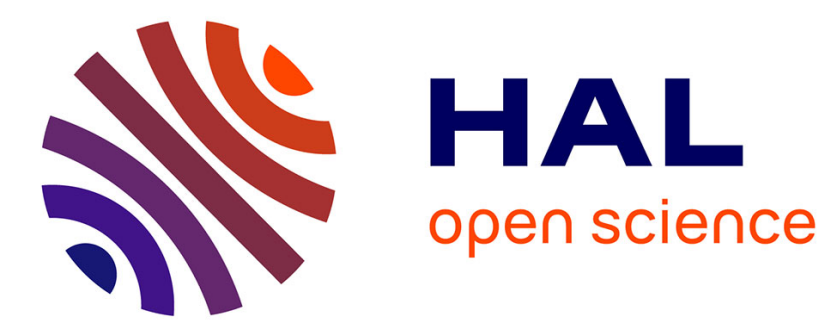

\title{
Coded random access for massive MTC under statistical channel knowledge
}

Diane Duchemin, Lélio Chetot, Jean-Marie Gorce, Claire Goursaud

\section{To cite this version:}

Diane Duchemin, Lélio Chetot, Jean-Marie Gorce, Claire Goursaud. Coded random access for massive MTC under statistical channel knowledge. SPAWC 2019 - 20th IEEE International Workshop on Signal Processing Advances in Wireless Communications, Jul 2019, Cannes, France. pp.1-5, 10.1109/SPAWC.2019.8815491 . hal-02153735

\section{HAL Id: hal-02153735 \\ https://hal.inria.fr/hal-02153735}

Submitted on 12 Jun 2019

HAL is a multi-disciplinary open access archive for the deposit and dissemination of scientific research documents, whether they are published or not. The documents may come from teaching and research institutions in France or abroad, or from public or private research centers.
L'archive ouverte pluridisciplinaire HAL, est destinée au dépôt et à la diffusion de documents scientifiques de niveau recherche, publiés ou non, émanant des établissements d'enseignement et de recherche français ou étrangers, des laboratoires publics ou privés. 


\title{
Coded random access for massive MTC under statistical channel knowledge
}

\author{
Diane Duchemin, Lélio Chetot, Jean-Marie Gorce and Claire Goursaud \\ CITI Laboratory \\ Univ Lyon, INRIA, INSA Lyon \\ Lyon, France \\ \{diane.duchemin, lelio.chetot, jean-marie.gorce, claire.goursaud\}@insa-lyon.fr
}

\begin{abstract}
This paper focuses on random uplink transmissions of a subset of nodes disseminated in a cell. Under the constraints of massive Machine Type Communication (mMTC) in cellular Low Power Wide Area Networks (LPWAN) and Ultra Reliable Low Latency Communications (URLLC), improving the capability of a receiver to detect simultaneously several transmissions with a high probability is important. Considering a very limited coordination between the receiver and the distributed transmitters, the usage of coded Non Orthogonal Multiple Access (NOMA) strategies is seducing. In this framework, we target synchronous direct data transmissions and propose an optimal detector of the active users with channel state information at the receiver limited to statistical knowledge. This algorithm is based on a Maximum Likelihood (ML) detector, under statistical channel knowledge only. We give the formulation of the optimal detector and we evaluate its performance, with different codelengths, code types (random Gaussian and Grassmannian codes) and for various number of antennas at the base station.

Index Terms-mMTC, URLLC, random access, statistical channel state information
\end{abstract}

\section{INTRODUCTION}

The recent trend for IoT communications brings the research community to look at new performance metrics thus broadening the scope of classical cellular networks [1, 2]. For instance, a strong interest is given to the the impact of massive access, short packets and capacity evaluation [3, 4, 5].

In the context of massive random access, this paper deals with the detection of simultaneous transmissions of short packets at a base station (BS) equipped with multiple antennas. The main objective is to lower the latency of short and concurrent uplink (UL) communications (either a short data transmission or a network access request) between a set of nodes and a BS. In addition, we want to avoid long and heavy handshake mechanisms, which would be too costly in terms of spectrum, energy or time consumption, while ensuring a reliable detection. Usual slotted ALOHA mechanisms exploit orthogonal resources to limit collisions between simultaneous requests but at the price of a resource efficiency loss.

Following the line of work proposed by Bockelmann et al. [4] which includes a focus on direct transmission, many propositions have been done to shorten the handshake mechanism.

This work has been funded by the French National Agency for Research (ANR) under grant $n^{\circ}$ ANR-16-CE25-0002 - EPHYL, and partly supported by the Inria-Nokia Bell Labs common labs under research action Network Information Theory
For instance, the reader is referred to the so-called one-stage and two-stages access, or to the 3GPP early data transmission scheme proposed for Cat-M or NB-IoT in Release 15 [6]. The latter allows to piggy-back a short data during the classical random access procedure, i.e. the data is transmitted at the third step instead of the fifth. However, as we do not consider the possibility to encapsulate pilot sequences in the transmission for channel estimation, we are also working under restrictedcoordination access assumptions. Indeed, any communication prior to the data or access request transmission would introduce high signalling overhead and would be irrelevant in the case of access request, since users willing to access the network would need to be already identified for allowing their channel estimation before they even send their access requests. For instance, in $[7,8,9]$ the channel is considered as a known parameter at the receiver. Having a method able to work under statistical Channel State Information (CSI) only, is therefore a harsh constraint we deal with in this paper.

To comply with numerous simultaneous access, the proposed multiple access strategy relies on a Non Orthogonal Multiple Access (NOMA) with either Gaussian [10] or Grassmannian [11] coding. It is worth mentioning that noncoordinated communication -in terms of CSI, as discussed in section II- is generally not considered in coded NOMA models. More specifically, we propose a multiple user detector for coded NOMA, with CSIR restricted to a statistical knowledge. In section II, we describe the model and we derive the related optimal detection rule in section III. Numerical results are provided in section IV, both for Grassmannian and Gaussian codes in the case of Single User Detection (SUD), and with Gaussian coding only for Multiple User Detection (MUD). Section $\mathrm{V}$ concludes the paper.

\section{MODELS DESCRIPTION}

Table I lists the mathematical notations as well as the main variables we use throughout the paper. $\mathbf{H}$ denotes a matrix; $\mathbf{h}$ a vector; $h$ a scalar variable and $H$ a constant.

The general setting is the following: a node set $\Phi$ is deployed around the access point, which we also denote base station (BS). We consider the UL traffic and assume that it is sporadic, i.e. a subset of $\Phi$ is active at a given time. Besides, a transmission occurs under the assumptions of a restricted coordination between the nodes and the $\mathrm{BS}$, and no coordination 
among the nodes. The limited coordination just enables the synchronisation of the UL messages at the receiver at the BS together with the codebook knowledge, and provides a power control feature such that, in average, the received power of all the nodes is identical. For instance, a broadcast beacon can be used by the active nodes to synchronise themselves and determine their relative path-loss so that they transmit their messages accordingly. In the following, the term "noncoordinated" refers to the CSI.

TABLE I: List of mathematical notations

\begin{tabular}{|cl|}
\hline Notation & Description \\
\hline$\|\cdot\|_{p}$ & $p$-norm \\
$|\cdot|$ & Modulus \\
$s$ & Complex valued scalar \\
$\boldsymbol{v}$ & Complex valued vector \\
$\boldsymbol{M}$ & Complex valued matrix \\
$t$ & Transpose of a matrix \\
$H$ & Conjugate transpose of a matrix \\
$\Phi$ & Set of nodes \\
$\phi$ & Total number of nodes \\
$\Omega$ & Active node(s) subset \\
$\Omega$ & Subset of $\Phi$ \\
$N$ & Cardinal of $\Omega$ \\
$A$ & Number of antennas at the BS \\
$M$ & Number of channel uses per code \\
$\boldsymbol{y}$ & Signal vector \\
$\boldsymbol{z}$ & Noise vector \\
$\boldsymbol{c}_{n}$ & Coding vector \\
$\boldsymbol{x}_{n}$ & Message vector \\
$\boldsymbol{h}_{a}$ & Channel vector \\
\hline
\end{tabular}

\section{A. Model with full CSIR}

Let first consider the case of a random access channel, where the nodes just want to request resources. Each node $n \in \Phi$ may transmit its own code $c_{n}$ (allocated in advance or randomly selected according to a specific procedure on which the BS and nodes agreed in advance). Following [8, 9], the expression of the signal received at the BS is :

$$
\boldsymbol{Y}=\left[\begin{array}{ccc}
y_{1,1} & \cdots & y_{1, M} \\
\vdots & \cdots & \vdots \\
y_{A, 1} & \cdots & y_{A, M}
\end{array}\right]=\sum_{n \in \underline{\Omega}} \sqrt{\rho} \boldsymbol{h}_{n} \boldsymbol{c}_{n}^{t}+\boldsymbol{Z}
$$

where $\boldsymbol{Y} \in \mathbb{C}^{A \times M}$ is the received signal over $M$ channel uses and on the $A$ antennas, $\rho$ is the received signal power and

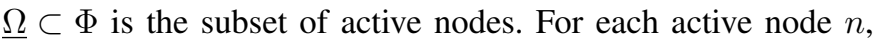
$\boldsymbol{c}_{n} \in \mathbb{C}^{M}$ is its code and, assuming a block and flat Rayleigh fading, independently distributed on each antenna of the base station, $\boldsymbol{h}_{n} \in \mathbb{C}^{A}$ is the channel impulse response associated to node $n . Z \in \mathbb{C}^{A \times M}$ represents the additive white Gaussian noise. Vectorizing (1) provides:

$$
\boldsymbol{y}=\sum_{n \in \underline{\Omega}} \sqrt{\rho} \mathbf{H}_{\mathbf{n}} \cdot \boldsymbol{c}_{n}+\boldsymbol{z}
$$

where $\mathbf{H}_{\mathbf{n}}=\mathbf{I}_{\mathbf{M}} \otimes \boldsymbol{h}_{n}$, with $\mathbf{I}_{\mathbf{M}}$ the identity matrix $M \times M$ and $\otimes$ the Kronecker product. We then have $\boldsymbol{y} \in \mathbb{C}^{A M}$ and $\boldsymbol{z} \in \mathbb{C}^{A M}$.

\section{B. Model with statistical CSIR}

In order to define a detector which is more suited for non-coordinated communications, we now adapt the former model, considering that the channel is unknown at the BS. Indeed, a full channel knowledge is not relevant with the non-coordination assumptions as it would require a previous communication where the users would be identified in order to deduce their channel impulse responses. Therefore, in our new scenario, the distribution of the channel responses is the only prior knowledge the BS possesses about the channel, not the actual realisations. The codes remain known. In this context, the signal can be reformulated as follow:

$$
\begin{aligned}
\boldsymbol{y} & =\sum_{n \in \underline{\Omega}} \sqrt{\rho}\left[\begin{array}{cccc}
\boldsymbol{c}_{n} & 0 & \ldots & 0 \\
0 & \boldsymbol{c}_{n} & \ddots & \vdots \\
\vdots & \ddots & \ddots & 0 \\
0 & \ldots & 0 & \boldsymbol{c}_{n}
\end{array}\right] \boldsymbol{h}_{n}+\boldsymbol{z} \\
& =\sum_{n \in \underline{\Omega}} \sqrt{\rho}\left(\mathbf{I}_{\mathbf{A}} \otimes \boldsymbol{c}_{n}\right) \boldsymbol{h}_{n}+\boldsymbol{z}
\end{aligned}
$$

In this new expression, the elements of $y$ are just reordered compared to eq. (1) such that $\boldsymbol{y}=\left[y_{1,1}, y_{1,2}, \ldots, y_{1, M}, y_{2,1}, \ldots, y_{A, 1}, \ldots, y_{A, M}\right]^{t}$ (i.e. we have vectorized the transpose of the matrix $\boldsymbol{Y}$ presented in (1)). The vectors $\boldsymbol{c}_{n}$ and $\mathbf{z}$ remain of the same nature: $\boldsymbol{c}_{n} \in \mathbb{C}^{M}$ is a given code and $\mathbf{z} \sim \mathcal{N}_{\mathbb{C}}\left(0, \mathbf{I}_{\mathrm{AM}}\right)$ while $\mathbf{h}_{\mathbf{n}} \sim \mathcal{N}_{\mathbb{C}}\left(0, \mathbf{I}_{\mathbf{A}}\right) \forall n \in \underline{\Omega}$.

\section{OPTIMAL DETECTOR}

We aim at minimizing the detection error i.e. $P[\Omega \neq \hat{\Omega}]$ where $\hat{\Omega}$ is the detected set and $\underline{\Omega}$ is the active subset thus, with $\Psi$ the set of all possible node subsets $\Omega$, we have:

$$
\begin{aligned}
\hat{\Omega} & =\underset{\Omega \in \Psi}{\arg \min } p[\underline{\Omega} \neq \Omega \mid \boldsymbol{y}] \\
& =\underset{\Omega \in \Psi}{\arg \max } p(\Omega \mid \boldsymbol{y}) \\
& =\underset{\Omega \in \Psi}{\arg \max } p(\boldsymbol{y} \mid \Omega) \frac{p(\Omega)}{p(\boldsymbol{y})} \\
& \stackrel{(i)}{=} \underset{\Omega \in \Psi}{\arg \max } p(\boldsymbol{y} \mid \Omega)
\end{aligned}
$$

(i) for an assumed uniform distribution of active sets.

Let $C_{\Omega}$ denote the matrix codeset of the nodes subset $\Omega$ :

$$
\boldsymbol{C}_{\Omega}=\left[\boldsymbol{c}_{1} ; \cdots ; \boldsymbol{c}_{n} ; \cdots \boldsymbol{c}_{N}\right]
$$

with $n \in \Omega$. Let its SVD be $\boldsymbol{C}_{\Omega}=\boldsymbol{V} \boldsymbol{\Lambda} \boldsymbol{U}$, where $\boldsymbol{V}$ and $\boldsymbol{U}$ are respectively $\mathbb{C}^{M \times M}$ and $\mathbb{C}^{N \times N}$ unitary matrices, and $\boldsymbol{\Lambda}$ is the $\mathbb{C}^{M \times N}$ matrix containing the singular values which we will denote $\lambda_{i}$. Note that $i \in[1 ; M]$ and $\lambda_{i}=0$ is possible in the cases where $M>N$. Let $\boldsymbol{y}_{a} \in \mathbb{C}^{M}$ be the received signal on antenna $a, \sigma \in \mathbb{C}^{M \times M}$ be given by:

$$
\sigma=\rho \boldsymbol{C}_{\Omega} \boldsymbol{C}_{\Omega}^{H}+\boldsymbol{I}_{\boldsymbol{M}}
$$


and

$$
\tilde{\boldsymbol{y}}_{a}=\operatorname{diag}\left(\sqrt{\frac{\rho\left|\lambda_{1}\right|^{2}}{1+\rho\left|\lambda_{1}\right|^{2}}}, \cdots, \sqrt{\frac{\rho\left|\lambda_{M}\right|^{2}}{1+\rho\left|\lambda_{M}\right|^{2}}}\right) \boldsymbol{V}^{\boldsymbol{H}} \boldsymbol{y}_{a}
$$

Proposition 1. Under the previously defined assumptions, the optimal Multi User Detector with statistical CSIR is:

$$
\hat{\Omega}=\underset{\Omega \in \Psi}{\arg \max } \prod_{a=1}^{A} \frac{1}{(2 \pi)^{M}|\sigma|} \exp \left(-\frac{1}{2}\left(\left\|\boldsymbol{y}_{a}\right\|_{2}^{2}-\left\|\tilde{\boldsymbol{y}}_{a}\right\|_{2}^{2}\right)\right)
$$

Proof. From eq.(3), given $\Omega$, we have:

$$
\boldsymbol{y}=\sqrt{\rho}\left[\begin{array}{lllll}
\boldsymbol{I}_{\boldsymbol{A}} \otimes \boldsymbol{c}_{1} & \cdots & \boldsymbol{I}_{\boldsymbol{A}} \otimes \boldsymbol{c}_{n} & \cdots & \boldsymbol{I}_{\boldsymbol{A}} \otimes \boldsymbol{c}_{N}
\end{array}\right] \boldsymbol{h}+\boldsymbol{z}
$$

where $\boldsymbol{h}=\left[\boldsymbol{h}_{1}^{t} \cdots \boldsymbol{h}_{n}^{t} \cdots \boldsymbol{h}_{N}^{t}\right]^{t}, n \in \Omega$. As $\boldsymbol{h}_{n}$ and $\boldsymbol{z}$ are both Gaussian, we thus have $\boldsymbol{y} \sim \mathscr{C} \mathcal{N}(0, \Sigma)$ and

$$
p(\boldsymbol{y} \mid \Omega)=\frac{1}{(2 \pi)^{A M}|\Sigma|} \exp \left(-\frac{1}{2} \boldsymbol{y}^{H} \Sigma^{-1} \boldsymbol{y}\right)
$$

with:

$$
\begin{aligned}
& \Sigma=\mathbb{E}\left[\boldsymbol{y} \cdot \boldsymbol{y}^{H}\right]
\end{aligned}
$$

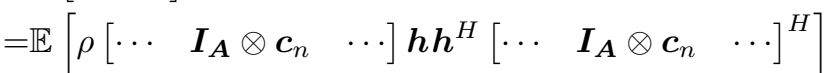

$$
\begin{aligned}
& +\mathbb{E}\left[\boldsymbol{z} \boldsymbol{z}^{H}\right] \\
& =\rho\left[\begin{array}{lll}
\cdots & \boldsymbol{I}_{\boldsymbol{A}} \otimes \boldsymbol{c}_{n} & \cdots
\end{array}\right]\left[\begin{array}{lll}
\cdots & \boldsymbol{I}_{\boldsymbol{A}} \otimes \boldsymbol{c}_{n} & \cdots
\end{array}\right]^{H}+\boldsymbol{I}_{\boldsymbol{A} \boldsymbol{M}} \\
& =\boldsymbol{I}_{\boldsymbol{A}} \otimes\left(\rho \boldsymbol{C}_{\Omega} \boldsymbol{C}_{\Omega}^{H}+\boldsymbol{I}_{\boldsymbol{M}}\right) \\
& =\boldsymbol{I}_{\boldsymbol{A}} \otimes\left(\rho \boldsymbol{V} \boldsymbol{\Lambda} \boldsymbol{\Lambda}^{H} \boldsymbol{V}^{H}+\boldsymbol{I}_{\boldsymbol{M}}\right) \\
& =\boldsymbol{I}_{\boldsymbol{A}} \otimes\left(\rho \boldsymbol{V} \boldsymbol{d i a g}\left(\left|\lambda_{1}\right|^{2}, \cdots,\left|\lambda_{M}\right|^{2}\right) \boldsymbol{V}^{H}+\boldsymbol{I}_{\boldsymbol{M}}\right)
\end{aligned}
$$

Thus, the determinant $|\Sigma|$ and the inverse of $\Sigma$ are respectively:

$$
\begin{aligned}
& |\Sigma|=\left|\boldsymbol{I}_{\boldsymbol{A}}\right|^{M} \cdot\left|\rho \boldsymbol{V} \boldsymbol{\Lambda} \boldsymbol{\Lambda}^{H} \boldsymbol{V}^{H}+\boldsymbol{I}_{\boldsymbol{M}}\right|^{A} \\
& =\prod_{a=1}^{A}\left|\rho \boldsymbol{V} \boldsymbol{\Lambda} \boldsymbol{\Lambda}^{H} \boldsymbol{V}^{H}+\boldsymbol{I}_{\boldsymbol{M}}\right| \\
& \Sigma^{-1}=\boldsymbol{I}_{\boldsymbol{A}} \otimes\left(\rho \boldsymbol{V} \boldsymbol{\Lambda} \boldsymbol{\Lambda}^{H} \boldsymbol{V}^{H}+\boldsymbol{I}_{\boldsymbol{M}}\right)^{-1} \\
& =\boldsymbol{I}_{\boldsymbol{A}} \otimes\left(\boldsymbol{V}\left(\rho \boldsymbol{\Lambda} \boldsymbol{\Lambda}^{H}+\boldsymbol{I}_{\boldsymbol{M}}\right)^{-1} \boldsymbol{V}^{H}\right) \\
& =\boldsymbol{I}_{\boldsymbol{A}} \otimes\left(\boldsymbol{V}\left[\begin{array}{ccc}
1-\frac{\rho\left|\lambda_{1}\right|^{2}}{1+\rho\left|\lambda_{1}\right|^{2}} & & 0 \\
& \ddots & \\
0 & & 1-\frac{\rho\left|\lambda_{M}\right|^{2}}{1+\rho\left|\lambda_{M}\right|^{2}}
\end{array}\right] \boldsymbol{V}^{\boldsymbol{H}}\right) \\
& =\boldsymbol{I}_{\boldsymbol{A}} \otimes\left(\boldsymbol{I}_{\boldsymbol{M}}-\boldsymbol{V} \boldsymbol{D} \boldsymbol{V}^{H}\right)
\end{aligned}
$$

where $D$ is a diagonal matrix with element $d_{i, i}=\frac{\rho\left|\lambda_{i}\right|^{2}}{1+\rho\left|\lambda_{i}\right|^{2}}$, $i \in[1, M]$ on its diagonal.

As a result, one can rewrite eq.(12) as the product of the pdf of the received signal on each antenna $a$ :

$$
\begin{aligned}
p(\boldsymbol{y} \mid \Omega) & =\prod_{a=1}^{A} \frac{1}{(2 \pi)^{M}|\sigma|} \exp \left(-\frac{1}{2} \boldsymbol{y}_{a}^{H}\left(\boldsymbol{I}_{\boldsymbol{M}}-\boldsymbol{V} \boldsymbol{D} \boldsymbol{V}^{H}\right) \boldsymbol{y}_{a}\right) \\
& =\prod_{a=1}^{A} \frac{1}{(2 \pi)^{M}|\sigma|} \exp \left(-\frac{1}{2}\left(\left\|\boldsymbol{y}_{a}\right\|_{2}^{2}-\left\|\tilde{\boldsymbol{y}}_{a}\right\|_{2}^{2}\right)\right)
\end{aligned}
$$

This result shows that the optimal detector consists in finding the codeset minimizing the squared chordal distance $\left\|\boldsymbol{y}_{a}\right\|_{2}^{2}-\left\|\tilde{\boldsymbol{y}}_{a}\right\|_{2}^{2}$ between the subspace spanned by the columns of $\boldsymbol{C}_{\Omega}$ and the observed signal $\boldsymbol{Y}$ (see [12] for more details). Indeed, when looking at (9), it is clear that $\tilde{\boldsymbol{y}}_{a}$ is the projection of $\boldsymbol{y}_{a}$ onto the subspace spanned by the rows of $\boldsymbol{V}$, which are nothing but the singular vectors for the subspace associated to the considered codeset.

In the following we propose to compare the performance of a receiver exploiting either the random Gaussian codes as in $[8,9]$ or the Grassmannian codes recently proposed in [11].

\section{NUMERICAL RESULTS}

In this section, we present numerical results of both SUD and MUD, obtained from the implementation of the optimal detector (10). It is worth mentioning that in these numerical results the number of users is limited to few units while a massive access would require thousands of users. Basically, the target of this paper is to evaluate the potential gain of using multi-user detection instead of a conventional Aloha access. To scale to larger user sets, this technique can be first extended to larger sets but at the price of a high computational complexity, except if an efficient algorithm could be derived. This aspect is out of the scope of this paper. The most straightforward option is to combine this multi-user technique with classical ones used for the massive access, e.g. slotted ALOHA as proposed in $5 \mathrm{G}$ standardization $[5,6]$.

The results are shown in terms of Codeset Error Rate (CER), i.e. the rate of detecting a subset $\hat{\Omega}$ while the active node subset is $\underline{\Omega}$, with $\hat{\Omega} \neq \underline{\Omega}$. For a reason of computational complexity at the receiver, several versions of the ML detector are provided, such as the ML with targeted search (TS), rather than the exhaustive search (ES), the latter corresponding to the optimal detector. The so-called "targeted search" means that the ML is performed only on the subsets of nodes $\Omega$ whose size $N$ equals that of the actual active subset $\underline{\Omega}$, assuming this side information is available at the receiver. It provides a lower bound on the CER. Moreover, the "iterative" (It) ML detector is an approximated ML algorithm as the detection is realised by accumulation of the detected users (one at each iteration), considering the detected users of the previous iterations as given for the current iteration. Its structure is the same as the proposed algorithm in [9]. If used together with the "targeted search" option, the iterations number thus matches the number of active nodes. To provide a reference, the CER of the ML with iterative targeted search and channel knowledge (labelled "full CSIR" in the figures) [9] is also given as the detection is performed with a full CSIR assumption rather than its restriction to statistical knowledge.

\section{A. SUD with Grassmannian codes vs Gaussian codes}

We first target a single user detection, meaning that we assume that one and only one user is active. As explained above we use two different codebooks. The codebook size is equal to 100 , and the codelength $M$ is chosen between 8 and 
16. The BS can be equipped with 4 or 8 antennas. We describe below the two coding techniques.

a) Random Gaussian codebook: Complex Gaussian random values are selected for each coefficient in the codebook matrix. Each code is then normalized to a unitary power. In the Monte-Carlo simulations, a new codebook is generated for each couple of codelength and antennas number.

b) Grassmannian codebook: We use the complex codebook construction proposed in [11]. We address here the problem of designing the binary representation of the Grassmannian. We want to build $\phi$ codes (one for each possible user in $\Phi)$. We choose to split them equally between the $M$ dimensions, called hereafter as facets. For a given number of channel uses $M$, we use $\left\lceil\log _{2}(M)\right\rceil$ bits to encode the facet in the Grassmannian space. A facet has $2 M-2$ real dimensions (or $M-1$ complex dimensions) which will be given each 1 bit. Since each facet must have $\lceil\phi / M\rceil$ codes, we wish to choose them as far as possible, meaning that the binary representation of the codes must have the greatest Hamming distance for codes with $2 M-2$ bits. To address this problem, we encode the $\left\lceil\log _{2} \phi / M\right\rceil$ most significant bits with the binary representation of the integers $n_{i}$ in $\llbracket 0,\lceil\phi / M\rceil-1 \rrbracket$ and complete the remaining least significant bits with the CRC of the most significant bits. The CRC polynomials have been chosen with respect to the value of $M$ and the minimum distance they grant between the codes, see Table II. The

TABLE II: CRC generator polynomials

\begin{tabular}{cl}
\hline Number of channel uses & Polynomials (bin. representation) [13] \\
\hline 4 & 1011 \\
8 & 10100110111 \\
16 & 1100101101111010101000100111 \\
\hline
\end{tabular}

results for SUD are given in Fig 1. The first general and expected result is the decrease of the CER as the SNR $\rho$, the antennas number $A$ and/or the codelength $M$ increase(s) and the fact that increasing the number of degrees of freedom via the codelength has a greater impact on the performance than the antennas number. A second and more interesting result is the evaluation of the loss of performance between an iterative ML detector which considers the channel known [9], and the statistical ML detector given by eq. (10). Indeed, for $A=4, M=8$ the ML with CSIR clearly outperforms the statistical CSIR-ML with both Gaussian and Grassmannian codes and keep this trend for increasing values of $A$ and $M$. This demonstrates the cost of a highly restricted coordination.

Finally, we give a word about the performance of Grassmannian codes with respect to the Gaussian codes. Grassmannian codes are designed to be non-coherent codes which means that they are robust to channel fading since the information is encoded into the direction of the codes. What is observed from fig. 1 is that the CER for Grassmannian coding performs generally less than the Gaussian coding. However, Grassmannian codes have the clear advantage to benefit from their structures due to the way they are built. A piece of explanation for the difference observed between the two coding techniques could be the following: for a given Gaussian codebook, the associated minimum chordal distance may be in average greater than the minimum distance that is obtained from the Grassmannian codebook. Hence, the probability of confusing between two codesets would be lower for the Gaussian coding. Far from questioning the construction of the codes given in [11], we thus point the fact that choosing a good design at the binary level is not straightforward for granting the codes to perform well.

\section{B. MUD with Gaussian codes}

To the best of our knowledge, as no structured design of Grassmannian codebook is available for multiple users scenario, the MUD has then been performed with Gaussian codebooks for either $\phi=10$ or 20 users, for fixed values of codelength and number of antennas: $M=8, A=4$. In these simulations, the active subset size $N$ is distributed according to a Poisson law $\mathcal{P}(p)$ with parameter $p=2$ or $p=4.4$ versions of the detector are used, including the exact implementation of the ML from (10) realizing the exhaustive search of the active subset for the case $\phi=10$ where the complexity of this detection algorithm is reasonable. The "targeted search" detector uses $N$ as side information and thus performs a targeted search based on the active subset cardinal. The iterative version of the ML reduces the complexity by computing the tests on a restricted set of hypotheses, since the considered node subsets are build specifically for the current iteration, based on the result of the previous steps. The last CER depicted is from the iterative ML with the targeted search feature but also the full CSIR assumption.

Let us focus on the CER in Fig. 2 and Fig. 3 which depicts the MUD performance in the scenario of respectively $\phi=$ 10 and 20 users among which the active subset cardinal is $N \sim \mathcal{P}(p)$. The plain lines represent the case where $p=2$ while the dashed line are related to the case $p=4$. Again, as intended, the increase of $\rho$ improves the reliability. Moreover, as both are conducted from iterative and targeted detection, the green curves with circles and the yellow ones with + can be compared to show the impact of the channel knowledge on the CER under an iterative search assumption. At low SNR, the restriction to a statistical CSIR is shown by the increase of the CER from the full CSIR in green to the yellow curves. However, at high SNR, the chordal distance based detection under statistical CSIR performs better. On the contrary, the full CSIR performance decreases due to false alarms. Indeed, opposite codes can be subject to strong channel, bringing the resulting received signal at a closer euclidean distance from another code in between rather than any of them. In statistical CSIR, the chordal distance based computation is less subject to errors since it is not sensitive to orthogonal contributions, with respect to the active code direction.

The iterative implementation is close to actual performance of native ML version, either in targeted or exhaustive search. It thus could be used to lower the complexity of evaluation and for deployment with low loss. A confirmation from further studies are however needed at higher $M, A, \phi$. 


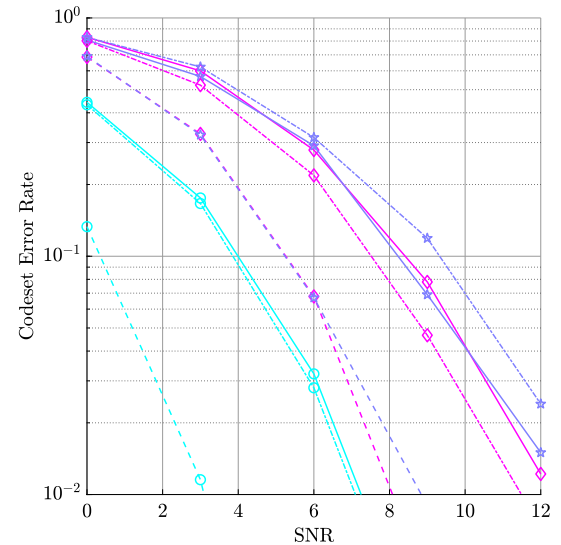

Fig. 1: CER under SUD ([_ ] $A=4, M=8$; [. - ] $A=4, M=16$; [-..] $A=8, M=8$; [ $\diamond$, purple] Gaussian ; [0, light blue] Gaussian with ItTS and full CSIR; [ dark blue] Grassmannian).

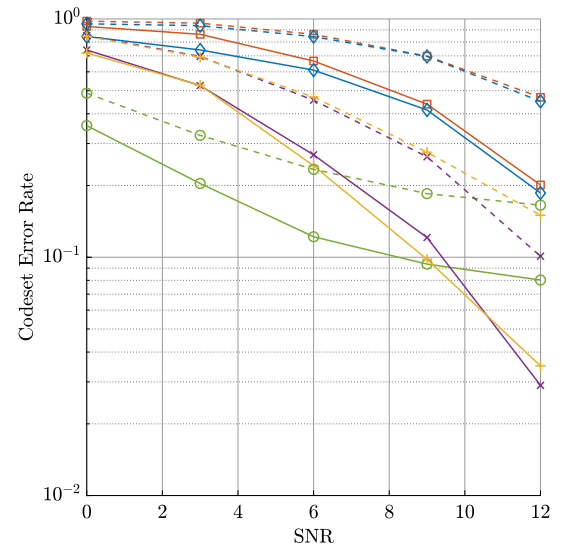

Fig. 2: CER under MUD with Gaussian coding for $\Phi=10$ users, $N \sim$ $\mathcal{P}(p)$ ([_] $p=2$; [- - ] $p=4$; [×, purple] TS; [+, yellow] It-TS; [ $\diamond$, blue] ES; [ $\square$, red] It-ES; [o , green] It-TS and full CSIR).

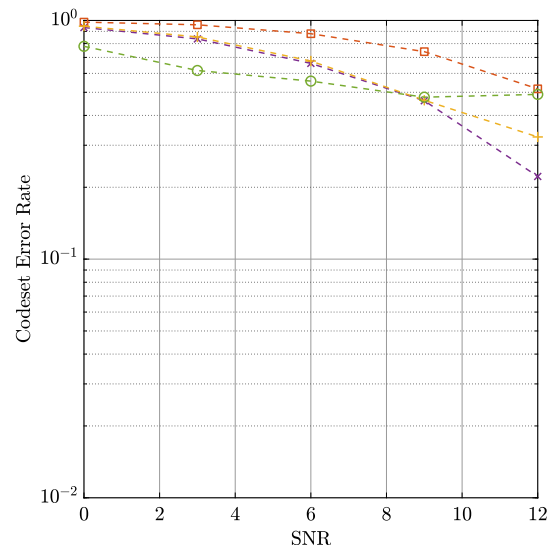

Fig. 3: CER under MUD with Gaussian coding for $\Phi=20$ users, $N \sim$ $\mathcal{P}(4)([\times$, purple] TS; [+, yellow $]$ ItTS; [ $\square$, red] It-ES; [o , green] It-TS and full CSIR).
We now compare the exhaustive search to the targeted search. We observe the obvious CER decrease by knowing the active subset size. Therefore, taking into account the complexity of the optimal detector (10), the estimation of the number of active nodes should provide a significant performance gain in statistical CSIR while reducing the required computational resources.

\section{CONCLUSION}

The paper proposes an optimal Multi User Detector for NOMA coded random access in a statistical CSIR scenario. The derivation of the optimal detector and the performance results are provided for MUD with multiple antennas at the BS. It gives an overview of the trade-offs between the system parameters (SNR, codelength and antennas number) which are relevant for URLLC as well as different detection policies. Furthermore, the detector shows that the chordal distance is still a valid metric for codeset dimension larger than one. It underlines the need of developing good and structured codes also taking into account multiple access scenarios. Working on and extending Grassmannian codes to meet those needs would be an interesting step forwards.

\section{REFERENCES}

[1] J. Sachs et al. "Adaptive 5G Low-Latency Communication for Tactile InternEt Services". In: Proc. IEEE 107.2 (Feb. 2019), pp. 325-349.

[2] G. Durisi, T. Koch, and P. Popovski. "Toward Massive, Ultra-reliable, and Low Latency Wireless Communication with Short Packets". In: Proc. IEEE 104.9 (2016), pp. 1711-1726.

[3] C. Goursaud and J.-M. Gorce. "Dedicated networks for IoT: PHY / MAC state of the art and challenges". In: EAI Endorsed Transactions on Internet of Things 1.1 (Oct. 2015).
[4] Carsten Bockelmann et al. "Towards Massive Connectivity Support for Scalable mMTC Communications in 5G Networks". In: IEEE Access 6 (2018).

[5] M. Alam and Q. Zhang. "A survey: Non-orthogonal multiple access with compressed sensing multiuser detection for mmtc". In: arXiv:1810.05422 (2018).

[6] 3GPP. Further NB-IoT enhancements (RP-171428). 2017.

[7] Mehmood Alam and Qi Zhang. "A Survey: NonOrthogonal Multiple Access with Compressed Sensing Multiuser Detection for mMTC". Oct. 2018.

[8] R. Xie et al. "Many Access for Small Packets Based on Precoding and Sparsity-Aware Recovery". In: IEEE Trans. Commun. 64.11 (Nov. 2016), pp. 4680-4694.

[9] D. Duchemin, J.-M. Gorce, and C. Goursaud. "Low complexity Detector for massive uplink random access with NOMA in IoT LPWA networks". In: IEEE WCNC, Marrakech, Morocco). Apr. 2019.

[10] A.K. Fletcher, S. Rangan, and V.K. Goyal. "On-Off Random Access Channels: A Compressed Sensing Framework". https://arxiv.org/pdf/0903.1022v2.pdf. 2009.

[11] A. Decurninge and M. Guillaud. "Cube-Split: Structured Quantizers on the Grassmannian of Lines". In: IEEE WCNC. Mar. 2017.

[12] J. H. Conway, R. H. Hardin, and N. J. A. Sloane. "Packing Lines, Planes, etc.: Packings in Grassmannian Space". In: arXiv:math/0208004 (July 31, 2002).

[13] P. Koopman. Best CRC Polynomials. URL: https://users. ece.cmu.edu/ koopman/crc/. 